Disconjugacy, regularity of multi-indexed rationally extended potentials, and Laguerre exceptional polynomials

Y. Grandati' and C. Quesne'

Citation: Journal of Mathematical Physics 54, 073512 (2013); doi: 10.1063/1.4815997

View online: http://dx.doi.org/10.1063/1.4815997

View Table of Contents: http://aip.scitation.org/toc/jmp/54/7

Published by the American Institute of Physics

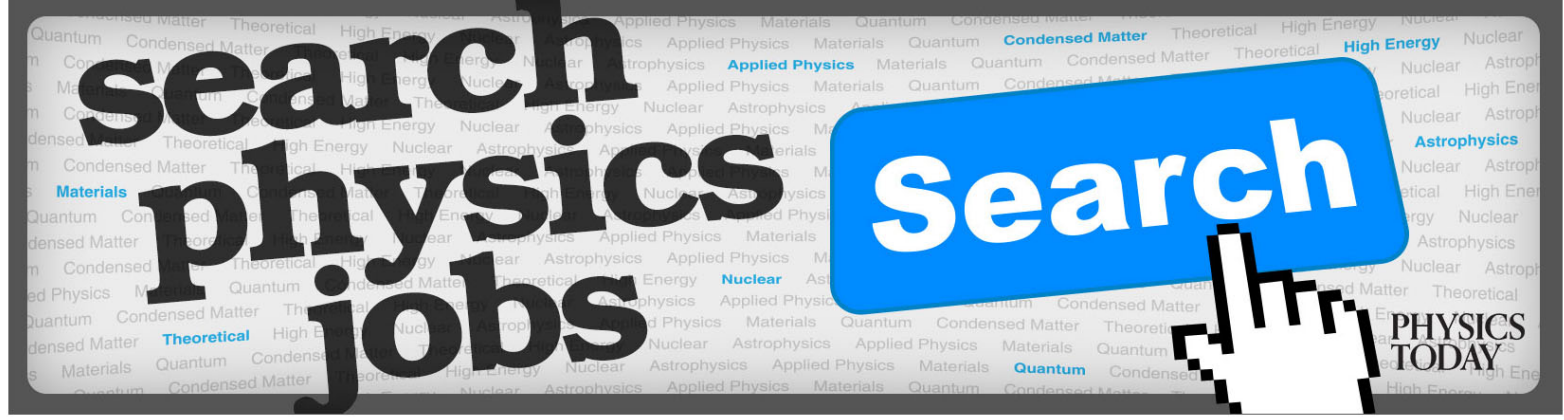




\title{
Disconjugacy, regularity of multi-indexed rationally extended potentials, and Laguerre exceptional polynomials
}

\author{
Y. Grandati ${ }^{1, a)}$ and C. Quesne ${ }^{2, b)}$ \\ ${ }^{1}$ Equipe BioPhysStat, LCP A2MC, Université de Lorraine-Site de Metz, 1 bvd D. F. Arago, \\ F-57070 Metz, France \\ ${ }^{2}$ Physique Nucléaire Théorique et Physique Mathématique, Université Libre de Bruxelles, \\ Campus de la Plaine CP229, Boulevard du Triomphe, B-1050 Brussels, Belgium
}

(Received 4 December 2012; accepted 4 July 2013; published online 25 July 2013)

\begin{abstract}
The power of the disconjugacy properties of second-order differential equations of Schrödinger type to check the regularity of rationally extended quantum potentials connected with exceptional orthogonal polynomials is illustrated by re-examining the extensions of the isotonic oscillator (or radial oscillator) potential derived in $k$ th-order supersymmetric quantum mechanics or multistep Darboux-Bäcklund transformation method. The function arising in the potential denominator is proved to be a polynomial with a nonvanishing constant term, whose value is calculated by induction over $k$. The sign of this term being the same as that of the already known highest degree term, the potential denominator has the same sign at both extremities of the definition interval, a property that is shared by the seed eigenfunction used in the potential construction. By virtue of disconjugacy, such a property implies the nodeless character of both the eigenfunction and the resulting potential. (C) 2013 AIP Publishing LLC. [http://dx.doi.org/10.1063/1.4815997]
\end{abstract}

\section{INTRODUCTION}

The introduction of the concept of exceptional orthogonal polynomials (EOP) $)^{1,2}$ and the construction of the first $X_{1}$ families of Laguerre- and Jacobi-type EOP in the context of Sturm-Liouville theory ${ }^{3,4}$ have aroused a lot of interest both in mathematics and in physics. On the one hand, the EOP families are novel systems of complete orthogonal polynomials with respect to some positivedefinite measure, generalizing the classical families of Hermite, Laguerre, and Jacobi, but differing from these among others by the presence of gaps in the degrees $n$ of the polynomials appearing in the sequences (e.g., $n=0$ for the $X_{1}$ families). On the other hand, they were shown ${ }^{5}$ to provide a keystone for rationally extending some well-known (translationally) shape-invariant quantum potentials ${ }^{6-8}$ in such a way that shape invariance is preserved. In this context, the usefulness of an approach based on supersymmetric quantum mechanics (SUSYQM) or, equivalently, the Darboux transformation was pointed out. ${ }^{9,10}$

An important step was the description of infinitely many shape-invariant potentials associated with $X_{m}$ EOP families, where the number of gaps $m$, called codimension (and corresponding here to $n=0,1, \ldots, m-1)$, may be arbitrarily large. ${ }^{11-14}$ The existence of two families of $X_{m}$-Laguerre and $X_{m}$-Jacobi EOP was also reported (thereby extending an observation made for $m=2$ in Ref. 10) and it was later on explained through Darboux-Crum transformation. ${ }^{15-17}$ In addition, these families were shown to be obtainable through two alternative approaches, namely, the Darboux-Bäcklund transformation (DBT) ${ }^{18}$ and the prepotential method. ${ }^{19}$

Counterparts of the classical family of Hermite polynomials have also been known in physical applications since the early $1990 \mathrm{~s},{ }^{20-27}$ but these $X_{m}$ EOP are of a slightly different nature in the

a) grandati@yahoo.fr
b) cquesne@ulb.ac.be 
sense that the $m$ gaps in the degree sequence correspond to $n=1,2, \ldots, m$, instead of $n=0,1, \ldots$, $m-1$. Since then, similar $X_{m}$-Laguerre and $X_{m}$-Jacobi EOP have been constructed and form the so-called third families. ${ }^{10,18,19}$

A significant advance was then the introduction of multi-indexed $X_{m_{1}, m_{2}, \ldots, m_{k}}$ EOP families and associated rationally extended potentials through multistep Darboux algebraic transformations. ${ }^{28}$ The same concept was developed through several approaches, such as the Crum-Adler mechanism, ${ }^{29}$ higher order SUSYQM, ${ }^{30,31}$ and multistep DBT. ${ }^{32}$

In such a context, there remain two open essential questions. The first one is to know whether the multi-indexed EOP families exhaust all the possibilities of higher codimensional complete orthogonal polynomial systems or, in other words, whether all the higher codimensional complete orthogonal polynomial systems are generated by the application of successive algebraic Darboux transformations (or one of the equivalent methods quoted above). This was recently proved to be true for codimension two. ${ }^{33}$

The second problem has to do with the existence of a well-behaved measure defining an EOP system, which is directly connected with the regularity of the associated rationally extended quantum potentials. In the one-indexed $X_{m}$ case, this problem is usually solved by showing that the classical polynomial occurring in the denominator has no zero in the domain of the variable, which is an easy task since the distribution of zeros of classical polynomials is well known. ${ }^{34,35}$ In the multi-indexed $X_{m_{1}, m_{2}, \ldots, m_{k}}$ case, however, the denominator contains a Wronskian of functions written in terms of classical polynomials, so that the problem becomes much more difficult to solve. While still feasible for $k=2$, the study of zeros remains outside scope for most higher $k$ cases, hence the construction of multi-indexed EOP is still rather formal.

A notable progress towards the solution of the regularity question was recently made ${ }^{32}$ by making use of the disconjugacy properties of the Schrödinger equation for eigenvalues below the ground state.$^{36-38}$ As a consequence of such properties, the nodeless character of the function present in the denominator can be inferred from the signs it takes on both ends of the domain. Nevertheless when applied to the rationally extended isotonic oscillator (or radial oscillator) potentials, ${ }^{32}$ except in the $k=2$ case, the equality of signs was obtained without exhibiting explicit formulas for the denominator in the asymptotic regions.

The purpose of the present paper is to give such explicit expressions in the general multi-indexed case, where each step may be associated with any one of the first two families of $X_{m}$-Laguerre EOP (although of interest the mixing with steps associated to the third family necessitates a separate analysis and is not considered in the present article). We then extend some results previously obtained in Refs. 30 and 31, confirming in particular a conjecture about the structure of the polynomial factor in the denominator. This allows a more direct proof of the regular character of the considered extensions.

The paper is organized as follows. After recalling in Sec. II the disconjugacy properties of the Schrödinger equation, we apply it to the construction of rationally extended radial oscillator potentials and corresponding $X_{m}$-Laguerre EOP in first-order SUSYQM. Section III summarizes the corresponding multistep construction in $k$ th-order SUSYQM. An improved proof of the regularity of these extensions is then given in Sec. IV. Finally, Sec. V contains the conclusion.

\section{RATIONAL EXTENSIONS OF THE ISOTONIC POTENTIAL AND $X_{m}$-LAGUERRE EOP}

\section{A. Disconjugacy}

Let us briefly recall some essential elements about the disconjugacy properties of second-order differential equations of Schrödinger type,

$$
\psi^{\prime \prime}(x)+(E-V(x)) \psi(x)=0 .
$$

To any solution $\psi(x)$ of Eq. (2.1) we can associate the corresponding Ricatti-Schrödinger function $w(x)=-\psi^{\prime}(x) / \psi(x)$, which is a solution of

$$
-w^{\prime}(x)+w^{2}(x)=V(x)-E .
$$


Equation (2.1) is said to be disconjugated on $I \subset \mathbb{R}(V(x)$ being supposed continuous on $I)$ if every solution of this equation has at most one (necessarily simple) zero on $I .^{36,37}$ For a closed or open interval $I$, the disconjugacy of Eq. (2.1) is equivalent to the existence of solutions that are everywhere nonzero on $I .^{36,37}$ In the following we will consider $\left.I=\right] 0,+\infty[$.

Let us also quote the following result:

Theorem (Refs. 36 and 37). If there exists a continuously differentiable solution on I of the Riccati inequality

$$
-w^{\prime}(x)+w^{2}(x) \leq V(x)-E
$$

then Eq. (2.1) is disconjugated on I.

In the case we consider, the Hamiltonian corresponding to Eq. (2.1) and associated to Dirichlet boundary conditions on $I$ has an infinite bound-state spectrum $\left\{E_{v}, \psi_{v}\right\}_{v \in \mathbb{N}}$. Consequently, since $\psi_{0}$ is nodeless on $I, w_{0}(x)$ is a continuously differentiable solution of (2.2) on this domain and condition (2.3) is fulfilled for every value of $E$ not greater than $E_{0}$. The domain of values ] $-\infty$, $\left.E_{0}\right]$ of the spectral parameter $E$ will be called the disconjugacy sector of Eq. (2.1). In this sector, except for $E=E_{0}, \psi(x)$ does not satisfy the required Dirichlet boundary conditions to be a boundstate wavefunction and diverges at one or both extremities of $I$. To ensure that this eigenfunction is nodeless, it is sufficient to verify that it has the same sign at both extremities of the definition interval.

\section{B. Isotonic oscillator and $\boldsymbol{X}_{\boldsymbol{m}}$-Laguerre EOP}

The isotonic oscillator potential is defined on the positive half-line $x>0$ as

$$
V_{l}(x)=\frac{1}{4} \omega^{2} x^{2}+\frac{l(l+1)}{x^{2}} .
$$

The spectrum of the associated Schrödinger equation has an infinite number of bound states, whose (unnormalized) wavefunctions are given by

$$
\psi_{v}^{(l)}(x) \propto \eta_{l}(z) L_{v}^{(\alpha)}(z), \quad v \in \mathbb{N},
$$

where $z=\omega x^{2} / 2, \alpha=l+1 / 2$, and

$$
\eta_{l}(z)=z^{(\alpha+1 / 2) / 2} e^{-z / 2},
$$

$L_{v}^{(\alpha)}(z)$ being a generalized Laguerre polynomial. ${ }^{34}$ The associated energies are

$$
E_{v}^{(l)}=\omega(2 v+\alpha+1) .
$$

In first-order SUSYQM, the superpartner Hamiltonians are defined as ${ }^{7}$

$$
\left\{\begin{array}{l}
H^{(+)}=A^{\dagger} A=-\frac{d^{2}}{d x^{2}}+V^{(+)}(x)-E, \\
H^{(-)}=A A^{\dagger}=-\frac{d^{2}}{d x^{2}}+V^{(-)}(x)-E,
\end{array}\right.
$$

with

$$
\left\{\begin{aligned}
A^{\dagger} & =-\frac{d}{d x}+W(x), \\
A & =\frac{d}{d x}+W(x),
\end{aligned}\right.
$$

where the superpotential $W(x)$ satisfies the Riccati equations

$$
\mp W^{\prime}(x)+W^{2}(x)=V^{( \pm)}(x)-E .
$$

$W(x)$ is obtained from a solution $\phi(x)$ of the initial Schrödinger equation

$$
H^{(+)} \phi(x)=0,
$$


via

$$
W(x)=-\phi^{\prime}(x) / \phi(x) .
$$

The new potential $V^{(-)}(x)=V^{(+)}(x)+2 W^{\prime}(x)$ is regular as soon as the "seed" solution $\phi(x)$ is nodeless on $] 0,+\infty$ [. This can be achieved if we choose the factorization energy in the disconjugacy sector of $H^{(+)}$, that is, when $E$ is not greater than the ground-state energy $E_{0}^{(+)}$of $V^{(+)}(x)$. This is true in particular if we choose as seed solution the ground-state wavefunction $\psi_{0}^{(l)}(x)=\eta_{l}(z)$ itself. For $E<E_{0}^{(+)}$, as discussed above, the analysis of the sign of $\phi(x)$ at $0^{+}$and $+\infty$ is then sufficient to control the regularity of $W(x)$.

Suppose that we are in this case. Then we have three possibilities (up to a global change of sign), namely,

$$
\left\{\begin{array}{cc}
\text { I }: \phi\left(0^{+}\right)=0^{+}, & \phi(+\infty)=+\infty, \\
\text { II }: \phi\left(0^{+}\right)=+\infty, & \phi(+\infty)=0^{+}, \\
\text {III }: \phi\left(0^{+}\right)=+\infty, & \phi(+\infty)=+\infty .
\end{array}\right.
$$

In the first two cases, $1 / \phi(x)$ diverges at one extremity of the positive half-line and, although we have

$$
H^{(-)}\left(\frac{1}{\phi(x)}\right)=0,
$$

it is not a bound-state wavefunction of $H^{(-)}$. Then $H^{(+)}$and $H^{(-)}$turn out to be isospectral. If we are looking for seed functions which are polynomials (up to some gauge factor), we obtain them from the eigenstates by applying the following discrete symmetries acting on the parameters of the initial potential (2.4): ${ }^{18}$

$$
\left\{\begin{array}{c}
\Gamma_{\mathrm{I}}:(\omega, \alpha) \rightarrow(-\omega, \alpha), \\
\Gamma_{\mathrm{II}}:(\omega, \alpha) \rightarrow(\omega,-\alpha), \\
\Gamma_{\mathrm{III}}:(\omega, \alpha) \rightarrow(-\omega,-\alpha) .
\end{array}\right.
$$

By applying the symmetry $\Gamma_{i}, i=\mathrm{I}$, II, III, to $\psi_{v}^{(l)}$, we obtain a seed eigenfunction $\phi_{\nu}$, which satisfies boundary conditions of type $i$ (see (2.13)). In particular, for $i=\mathrm{I}$ or II, we obtain

$$
\left\{\begin{array}{l}
\Gamma_{\mathrm{I}}: \psi_{m}^{(l)}(x) \rightarrow \phi_{l m}^{\mathrm{I}}(x)=\chi_{l}^{\mathrm{I}}(z) L_{m}^{(\alpha)}(-z), \\
\Gamma_{\mathrm{II}}: \psi_{m}^{(l)}(x) \rightarrow \phi_{l m}^{\mathrm{II}}(x)=\chi_{l}^{\mathrm{II}}(z) L_{m}^{(-\alpha)}(z),
\end{array}\right.
$$

where

$$
\left\{\begin{array}{c}
\chi_{l}^{\mathrm{I}}(z)=z^{(\alpha+1 / 2) / 2} e^{z / 2} \\
\chi_{l}^{\mathrm{II}}(z)=z^{-(\alpha-1 / 2) / 2} e^{-z / 2}
\end{array}\right.
$$

for the corresponding energies

$$
\left\{\begin{array}{l}
E_{l m}^{\mathrm{I}}=-\omega(\alpha+2 m+1) \\
E_{l m}^{\mathrm{II}}=-\omega(\alpha-2 m-1)
\end{array}\right.
$$

If $\phi_{l m}^{\mathrm{I}}$ is always in the disconjugacy sector, for $\phi_{l m}^{\mathrm{II}}$ to reach it we need to assume the condition $\alpha>m$. Starting from the isotonic potential $V^{(+)}(x)=V_{l^{\prime}}(x)$, the SUSYQM partnership based on seed eigenfunctions $\phi_{l^{\prime} m}^{\mathrm{I}}$ or $\phi_{l^{\prime} m}^{\mathrm{II}}$ with, respectively, $l^{\prime}=l-1$ and $l^{\prime}=l+1$ gives then rational extensions of the form ${ }^{31}$

$$
V^{(-)}(x)=V_{l}(x)+V_{l, \text { rat }}(x)+C,
$$


where

$$
V_{l, \mathrm{rat}}(x)=-2 \omega\left\{\frac{\dot{g}_{m}^{(\alpha)}}{g_{m}^{(\alpha)}}+2 z\left[\frac{\ddot{g}_{m}^{(\alpha)}}{g_{m}^{(\alpha)}}-\left(\frac{\dot{g}_{m}^{(\alpha)}}{g_{m}^{(\alpha)}}\right)^{2}\right]\right\}
$$

(the dot denoting a derivative with respect to $z=\omega x^{2} / 2$ ), with in the first case

$$
g_{m}^{(\alpha)}(z)=L_{m}^{(\alpha-1)}(-z), \quad C=-\omega
$$

and in the second case

$$
g_{m}^{(\alpha)}(z)=L_{m}^{(-\alpha-1)}(z), \quad C=\omega .
$$

Due to disconjugacy properties, the regularity of these extensions on the positive half-line is ensured for every $m=1,2, \ldots$ (and $\alpha$ large enough for type II).

The bound-state wavefunctions of the superpartner potential $V^{(-)}(x)$ are obtained by applying the operator $A$ (with $W(x)=-\phi_{l^{\prime} m}^{i \prime}(x) / \phi_{l^{\prime} m}^{i}(x), i=\mathrm{I}$, II) on those of $V^{(+)}(x)\left(\psi_{v}^{(+)}(x) \propto \eta_{l^{\prime}}(z) L_{v}^{\left(\alpha^{\prime}\right)}(z)\right.$ with $\left.\alpha^{\prime}=l^{\prime}+1 / 2\right)$. We can write

$$
\psi_{v}^{(-)}(x) \propto \frac{\eta_{l}(z)}{g_{m}^{(\alpha)}(z)} y_{m+v}^{(\alpha)}(z), \quad v=0,1,2, \ldots,
$$

where $y_{m+v}^{(\alpha)}(z)$ is a polynomial of degree $n=m+v$,

$$
y_{n}^{(\alpha)}(z)=\left\{\begin{array}{l}
L_{\alpha, m, n}^{\mathrm{I}}(z) \text { if } i=\mathrm{I}, \\
L_{\alpha, m, n}^{\mathrm{II}}(z) \text { if } i=\mathrm{II},
\end{array}\right.
$$

satisfying the second-order differential equation

$$
\left[z \frac{d^{2}}{d z^{2}}+\left(\alpha+1-z-2 z \frac{\dot{g}_{m}^{(\alpha)}}{g_{m}^{(\alpha)}}\right) \frac{d}{d z}+(z-\alpha) \frac{\dot{g}_{m}^{(\alpha)}}{g_{m}^{(\alpha)}}+z \frac{\ddot{g}_{m}^{(\alpha)}}{g_{m}^{(\alpha)}}\right] y_{m+v}^{(\alpha)}(z)=-v y_{m+v}^{(\alpha)}(z) .
$$

The set of polynomials $y_{m+v}^{(\alpha)}(z)(v=0,1,2, \ldots)$, defined on the positive half-line, is orthogonal and complete with respect to the positive-definite measure of density

$$
z^{\alpha} e^{-z} /\left(g_{m}^{(\alpha)}(z)\right)^{2}
$$

\section{LAGUERRE EOP AND REDUCIBLE $k$ TH ORDER SUSYQM}

In $k$ th-order SUSYQM, the first-order differential operators $A$ and $A^{\dagger}$ are replaced by $k$ th-order ones $\mathcal{A}$ and $\mathcal{A}^{\dagger} \cdot{ }^{22,23,39-41}$ The correspondence between the initial and final Hamiltonians $H^{(1)}$ and $H^{(2)}$ is determined by the intertwining relations

$$
\mathcal{A} H^{(1)}=H^{(2)} \mathcal{A}, \quad \mathcal{A}^{\dagger} H^{(2)}=H^{(1)} \mathcal{A}^{\dagger} .
$$

In the reducible case, the operators $\mathcal{A}$ and $\mathcal{A}^{\dagger}$ can be factorized into products of first-order differential operators as

$$
\mathcal{A}=A^{(k)} A^{(k-1)} \cdots A^{(1)},
$$

where

$$
A^{(i)}=\frac{d}{d x}+W^{(i)}(x), \quad W^{(i)}(x)=-\left(\log \phi^{(i)}(x)\right)^{\prime},
$$

the seed eigenfunctions being given by Crum's formula ${ }^{42}$

$$
\phi^{(i)}(x)=\phi_{1,2, \ldots, i}^{(i)}(x)=\frac{\mathcal{W}\left(\phi_{1}, \phi_{2}, \ldots, \phi_{i} \mid x\right)}{\mathcal{W}\left(\phi_{1}, \phi_{2}, \ldots, \phi_{i-1} \mid x\right)},
$$


with $\mathcal{W}\left(\phi_{1}, \phi_{2}, \ldots, \phi_{i} \mid x\right)$ denoting the Wronskian of $\phi_{1}(x), \phi_{2}(x), \ldots, \phi_{i}(x)$. Here we have introduced lower indices $1,2, \ldots, i$ to specify which seed functions have been used. The partner potentials are then linked by the second Crum's formula

$$
V^{(2)}(x)=V^{(1)}(x)-2 \frac{d^{2}}{d x^{2}} \log \mathcal{W}\left(\phi_{1}, \phi_{2}, \ldots, \phi_{k} \mid x\right)
$$

As in Sec. II, we start from the isotonic potential $V^{(1)}(x)=V_{l^{\prime}}(x)$ to get an extended potential of the form

$$
V^{(2)}(x)=V_{l}(x)+V_{l, \text { rat }}(x)+C
$$

The final potential is of type $\mathrm{I}^{q} \mathrm{II}^{k-q}$ if the chosen set of seed eigenfunctions includes $q \leq k$ seed functions of type I and $k-q$ seed functions of type II. The order of the seed functions being irrelevant, we can assume that $\left(z=\omega x^{2} / 2\right)$

$$
\phi_{i}(x)=\left\{\begin{array}{l}
\phi_{l^{\prime} m_{i}}^{\mathrm{I}}(x)=\chi_{l^{\prime}}^{\mathrm{I}}(z) L_{m_{i}}^{\left(\alpha^{\prime}\right)}(-z), i=1, \ldots, q, \\
\phi_{l^{\prime} m_{i}}^{\mathrm{II}}(x)=\chi_{l^{\prime}}^{\mathrm{II}}(z) L_{m_{i}}^{\left(-\alpha^{\prime}\right)}(z), i=q+1, \ldots, k
\end{array}\right.
$$

with $l^{\prime}=l+k-2 q$ (hence $\alpha^{\prime}=\alpha+k-2 q$ ), $0<m_{1}<\cdots<m_{q}$, and $0<m_{q+1}<\cdots$ $<m_{k}$, these functions being nodeless for $\alpha^{\prime}>\sup _{i=q+1, \ldots, k}\left(m_{i}\right)$. In Ref. 31, it has been shown that the Wronskian appearing in Crum's formula (3.5) can be written as

$$
\mathcal{W}\left(\phi_{1}, \phi_{2}, \ldots, \phi_{k} \mid x\right)=(2 \omega z)^{k(k-1) / 4} z^{-q(k-q)}\left(\chi_{l^{\prime}}^{\mathrm{I}}\right)^{q}\left(\chi_{l^{\prime}}^{\mathrm{II}}\right)^{k-q} g_{\mu}^{(\alpha)}(z)
$$

where

$$
g_{\mu}^{(\alpha)}(z)=z^{-(k-q)(k-q-1)} \operatorname{det} \tilde{\Gamma}_{\mu}^{(\alpha)}
$$

with

$$
\left(\tilde{\Gamma}_{\mu}^{(\alpha)}\right)_{i j}= \begin{cases}L_{m_{j}-i+1}^{\left(\alpha^{\prime}+i-1\right)}(-z), & 1 \leq i \leq q+1,1 \leq j \leq q, \\ \left(m_{j}+1\right)_{i-1} z^{k-i} L_{m_{j}+i-1}^{\left(-\alpha^{\prime}-i+1\right)}(z), & 1 \leq i \leq q+1, q+1 \leq j \leq k, \\ L_{m_{j}-q}^{\left(\alpha^{\prime}+i-1\right)}(-z), & q+2 \leq i \leq k, 1 \leq j \leq q, \\ \left(m_{j}+1\right)_{q}\left(m_{j}-\alpha^{\prime}-i+q+2\right)_{i-q-1} & \\ \times z^{k-i} L_{m_{j}+q}^{\left(-\alpha^{\prime}-i+1\right)}(z), & q+2 \leq i \leq k, q+1 \leq j \leq k,\end{cases}
$$

and $(a)_{n}=a(a+1) \cdots(a+n-1)$ denoting the usual Pochhammer symbol. ${ }^{34}$

Observe that on taking (3.5) and (3.8) into account, we can express $V_{l, \text { rat }}(x)$ and $C$ of Eq. (3.6) as

$$
V_{l, \text { rat }}(x)=-2 \omega\left\{\frac{\dot{g}_{\mu}^{(\alpha)}}{g_{\mu}^{(\alpha)}}+2 z\left[\frac{\ddot{g}_{\mu}^{(\alpha)}}{g_{\mu}^{(\alpha)}}-\left(\frac{\dot{g}_{\mu}^{\alpha)}}{g_{\mu}^{(\alpha)}}\right)^{2}\right]\right\}, \quad C=(k-2 q) \omega .
$$

In Ref. 31, it has been proved that

$$
g_{\mu}^{(\alpha)}(z)=\mathcal{C}_{\mu}^{(\alpha)} z^{\mu}+\text { lower order terms, }
$$

with

$$
\left\{\begin{array}{c}
\mu=\sum_{i=1}^{k} m_{i}-q(q-1) / 2-(k-q)(k-q-1) / 2+q(k-q) \\
\mathcal{C}_{\mu}^{(\alpha)}=(-1)^{\sigma} \Delta\left(m_{1}, \ldots, m_{q}\right) \Delta\left(m_{q+1}, \ldots, m_{k}\right) /\left(m_{1} ! \ldots m_{k} !\right) \\
\sigma=\sum_{i=q+1}^{k} m_{i}+q(k-q)
\end{array}\right.
$$


$\Delta\left(n_{1}, \ldots, n_{l}\right)$ being a Vandermonde determinant of order $l$,

$$
\Delta\left(n_{1}, \ldots, n_{l}\right)=\prod_{1 \leq i<j \leq l}\left(n_{j}-n_{i}\right) .
$$

As shown in Ref. 32, the Wronskian (3.8) has no node on the positive half-line, which ensures the regularity of the rationally extended potential $V^{(2)}(x)$ on this domain. The bound-state wavefunctions of the latter are then given by ${ }^{31}$

$$
\psi_{\nu}^{(2)}(x)=\frac{\eta_{l}(z)}{g_{\mu}^{(\alpha)}(z)} y_{\mu+v}^{(\alpha)}(z), \quad v=0,1,2, \ldots,
$$

where $y_{\mu+v}^{(\alpha)}(z)$ is a polynomial of degree $n=\mu+v$ satisfying the second-order differential equation

$$
\left[z \frac{d^{2}}{d z^{2}}+\left(\alpha+1-z-2 z \frac{\dot{g}_{\mu}^{(\alpha)}}{g_{\mu}^{(\alpha)}}\right) \frac{d}{d z}+(z-\alpha) \frac{\dot{g}_{\mu}^{(\alpha)}}{g_{\mu}^{(\alpha)}}+z \frac{\ddot{g}_{\mu}^{(\alpha)}}{g_{\mu}^{(\alpha)}}\right] y_{\mu+v}^{(\alpha)}(z)=-v y_{\mu+v}^{(\alpha)}(z) .
$$

The $y_{\mu+v}^{(\alpha)}(v=0,1,2, \ldots)$ polynomials constitute a complete orthogonal family on the positive half-line with respect to the positive-definite measure of density

$$
z^{\alpha} e^{-z} /\left(g_{\mu}^{(\alpha)}(z)\right)^{2}
$$

\section{POLYNOMIAL CHARACTER OF $\boldsymbol{g}_{\mu}^{(\alpha)}$ AND BEHAVIOUR NEAR THE ORIGIN}

In Ref. 31 it has been conjectured that $g_{\mu}^{(\alpha)}(z)$ is a polynomial, which can be directly checked in the $k=2$ case, but is not obvious for higher $k$ values whenever $k-q \geq 2$ (see Eq. (3.9)). In the following we will give a complete proof of this assertion. In Ref. 32 the regularity of the extended potential, which is equivalent to the absence of node of $g_{\mu}^{(\alpha)}$, has been established without giving the exact highest and lowest degrees coefficients of this polynomial. We will also determine the value of the lowest degree coefficient and then verify explicitly the nodeless character of the Crum Wronskian.

First note that from Eq. (3.8) we can write for $k-q \geq 2$

$$
\left\{\begin{array}{c}
\mathcal{W}\left(\phi_{1}, \phi_{2}, \ldots, \phi_{k} \mid x\right)=(2 \omega z)^{k(k-1) / 4} z^{-q(k-q)}\left(\chi_{l^{\prime}}^{\mathrm{I}}\right)^{q}\left(\chi_{l^{\prime}}^{\mathrm{II}}\right)^{k-q} g_{\mu}^{(\alpha)}(z), \\
\mathcal{W}\left(\phi_{1}, \phi_{2}, \ldots, \phi_{k-1} \mid x\right)=(2 \omega z)^{(k-1)(k-2) / 4} z^{-q(k-q-1)}\left(\chi_{l^{\prime}}\right)^{q}\left(\chi_{l^{\prime}}^{\mathrm{II}}\right)^{k-q-1} g_{\mu^{\prime}}^{(\alpha+1)}(z),
\end{array}\right.
$$

where $l^{\prime}=l+k-2 q$ (hence $\alpha^{\prime}=\alpha+k-2 q$ ) and

$$
\left\{\begin{aligned}
\mu & =\sum_{i=1}^{k} m_{i}-q(q-1) / 2-(k-q)(k-q-1) / 2+q(k-q), \\
\mu^{\prime} & =\sum_{i=1}^{k-1} m_{i}-q(q-1) / 2-(k-q-1)(k-q-2) / 2+q(k-q-1) \\
& =\mu-m_{k}+k-2 q-1 .
\end{aligned}\right.
$$

Inserting these expressions in Eq. (3.4), we arrive at

$$
\begin{aligned}
\phi_{1,2, \ldots, k}^{(k)}(x) & =(2 \omega z)^{(k-1) / 2} z^{-q} \chi_{l^{\prime}}^{\mathrm{II}}(z) \frac{g_{\mu}^{(\alpha)}(z)}{g_{\mu^{\prime}}^{(\alpha+1)}(z)} \\
& =(2 \omega)^{(k-1) / 2} z^{-\left(2 \alpha^{\prime}-2 k+4 q+1\right) / 4} e^{-z / 2} \frac{g_{\mu}^{(\alpha)}(z)}{g_{\mu^{\prime}}^{(\alpha+1)}(z)} .
\end{aligned}
$$

On the other hand, we can obtain a different expression for $\phi_{1,2, \ldots, k}^{(k)}$ by using some properties of Wronskians. Indeed, from Sylvester's theorem, ${ }^{43}$ we can write

$$
\mathcal{W}\left(\phi_{1}, \ldots, \phi_{k-1}, \phi_{k} \mid x\right)=\frac{\mathcal{W}\left(\mathcal{W}\left(\phi_{1}, \ldots, \phi_{k-2}, \phi_{k-1} \mid x\right), \mathcal{W}\left(\phi_{1}, \ldots, \phi_{k-2}, \phi_{k} \mid x\right) \mid x\right)}{\mathcal{W}\left(\phi_{1}, \ldots, \phi_{k-2} \mid x\right)}
$$


and using

$$
\mathcal{W}\left(f \phi_{1}, \ldots, f \phi_{k} \mid x\right)=f^{k} \mathcal{W}\left(\phi_{1}, \ldots, \phi_{k} \mid x\right)
$$

we have

$$
\begin{aligned}
& \mathcal{W}\left(\phi_{1}, \ldots, \phi_{k-1}, \phi_{k} \mid x\right)=\mathcal{W}\left(\phi_{1}, \ldots, \phi_{k-2} \mid x\right) \\
& \times \mathcal{W}\left(\frac{\mathcal{W}\left(\phi_{1}, \ldots, \phi_{k-2}, \phi_{k-1} \mid x\right)}{\mathcal{W}\left(\phi_{1}, \ldots, \phi_{k-2} \mid x\right)}, \frac{\mathcal{W}\left(\phi_{1}, \ldots, \phi_{k-2}, \phi_{k} \mid x\right)}{\mathcal{W}\left(\phi_{1}, \ldots, \phi_{k-2} \mid x\right)} \mid x\right),
\end{aligned}
$$

that is

$$
\phi_{1,2, \ldots, k}^{(k)}(x)=\frac{\mathcal{W}\left(\phi_{1, \ldots, k-2, k-1}^{(k-1)}, \phi_{1, \ldots, k-2, k}^{(k-1)} \mid x\right)}{\phi_{1, \ldots, k-2, k-1}^{(k-1)}(x)} .
$$

From the Wronskian theorem, ${ }^{44}$ it follows that the Wronskian in the numerator of this expression satisfies the property

$$
\left[\mathcal{W}\left(\phi_{1, \ldots, k-2, k-1}^{(k-1)}, \phi_{1, \ldots, k-2, k}^{(k-1)} \mid \xi\right)\right]_{x}^{+\infty}=\left(E_{k-1}-E_{k}\right) \int_{x}^{+\infty} \phi_{1, \ldots, k-2, k-1}^{(k-1)}(\xi) \phi_{1, \ldots, k-2, k}^{(k-1)}(\xi) d \xi
$$

because $\phi_{1, \ldots, k-2, k-1}^{(k-1)}$ and $\phi_{1, \ldots, k-2, k}^{(k-1)}$ correspond to the energies $\sum_{i=1}^{k-1} E_{i}$ and $\sum_{i=1}^{k-2} E_{i}+E_{k}$, respectively.

As a consequence of the exponential factor in Eq. (4.3), both $\phi_{1, \ldots, k-2, k-1}^{(k-1)}(\xi)$ and $\phi_{1, \ldots, k-2, k}^{(k-1)}(\xi)$ vanish for $\xi \rightarrow \infty$, which ensures the vanishing of their Wronskian in the same limit. Hence Eq. (4.8) reduces to

$$
\mathcal{W}\left(\phi_{1, \ldots, k-2, k-1}^{(k-1)}, \phi_{1, \ldots, k-2, k}^{(k-1)} \mid x\right)=-\left(E_{k-1}-E_{k}\right) \int_{x}^{+\infty} \phi_{1, \ldots, k-2, k-1}^{(k-1)}(\xi) \phi_{1, \ldots, k-2, k}^{(k-1)}(\xi) d \xi,
$$

or, on taking Eq. (4.3) into account and defining $\zeta=\omega \xi^{2} / 2$,

$$
\begin{aligned}
& \mathcal{W}\left(\phi_{1, \ldots, k-2, k-1}^{(k-1)}, \phi_{1, \ldots, k-2, k}^{(k-1)} \mid x\right)=-(2 \omega)^{k-5 / 2}\left(E_{k-1}-E_{k}\right) \\
& \times \int_{z}^{+\infty} \zeta^{k-2 q-\alpha^{\prime}-2} e^{-\zeta} \frac{g_{\mu^{\prime}}^{(\alpha+1)}(\zeta) g_{\bar{\mu}^{\prime}}^{(\alpha+1)}(\zeta)}{\left(g_{\mu^{\prime \prime}}^{(\alpha+2)}(\zeta)\right)^{2}} d \zeta
\end{aligned}
$$

where

$$
\left\{\begin{array}{c}
\bar{\mu}^{\prime}=\mu-m_{k-1}+k-2 q-1, \\
\mu^{\prime \prime}=\mu-m_{k-1}-m_{k}+2(k-2 q)-3 .
\end{array}\right.
$$

Inserting Eqs. (4.3) and (4.10) in the right-hand side of Eq. (4.7), we get the search for second expression of $\phi_{1,2, \ldots, k}^{(k)}(x)$,

$$
\begin{aligned}
\phi_{1,2, \ldots, k}^{(k)}(x)= & -(2 \omega)^{(k-3) / 2}\left(E_{k-1}-E_{k}\right) \\
& \times \int_{z}^{+\infty} \zeta^{k-2 q-\alpha^{\prime}-2} e^{-\zeta} g_{\mu^{\prime}}^{(\alpha+1)}(\zeta) g_{\bar{\mu}^{\prime}}^{(\alpha+1)}(\zeta) /\left(g_{\mu^{\prime \prime}}^{(\alpha+2)}(\zeta)\right)^{2} d \zeta \\
& \times\left[z^{\left(2 k-4 q-2 \alpha^{\prime}-3\right) / 4} e^{-z / 2} g_{\mu^{\prime}}^{(\alpha+1)}(z) / g_{\mu^{\prime \prime}}^{(\alpha+2)}(z)\right]^{-1},
\end{aligned}
$$

which can also be rewritten as a recursion relation for the $g_{\mu}^{(\alpha)}(z)$ functions,

$$
\begin{aligned}
g_{\mu}^{(\alpha)}(z)= & \frac{E_{k}-E_{k-1}}{2 \omega} z^{-\left(k-2 q-\alpha^{\prime}-1\right)} e^{z} g_{\mu^{\prime \prime}}^{(\alpha+2)}(z) \\
& \times \int_{z}^{+\infty} \zeta^{k-2 q-\alpha^{\prime}-2} e^{-\zeta} g_{\mu^{\prime}}^{(\alpha+1)}(\zeta) g_{\bar{\mu}^{\prime}}^{(\alpha+1)}(\zeta) /\left(g_{\mu^{\prime \prime}}^{(\alpha+2)}(\zeta)\right)^{2} d \zeta .
\end{aligned}
$$


On the right-hand side of this relation, $g_{\mu^{\prime}}^{(\alpha+1)}$ and $g_{\bar{\mu}^{\prime}}^{(\alpha+1)}$ correspond to $k^{\prime}=k-1$, while for $g_{\mu^{\prime \prime}}^{(\alpha+2)}, k^{\prime}=k-2$. The polynomial character of $g_{\mu}^{(\alpha)}(z)$ can be easily obtained by induction. This is already verified for $k=1$ and $k=2$. Assuming that for $k^{\prime}<k, g_{\mu}^{(\alpha)}$ is a polynomial with a non-zero constant term, it turns out that in the neighbourhood of the origin, the right-hand side of Eq. (4.13) has a well-defined value given by

$$
g_{\mu}^{(\alpha)}(z) \simeq \frac{m_{k}-m_{k-1}}{\alpha^{\prime}-k+2 q+1} g_{\mu^{\prime}}^{(\alpha+1)}(0) g_{\bar{\mu}^{\prime}}^{(\alpha+1)}(0) / g_{\mu^{\prime \prime}}^{(\alpha+2)}(0)(1+O(z)),
$$

where we have used $E_{k}=-\omega\left(\alpha^{\prime}-2 m_{k}-1\right)$.

From Eq. (4.14) we immediately deduce that $g_{\mu}^{(\alpha)}(z)$ cannot have a pole at the origin and is then a polynomial with non-zero constant term.

To completely describe the behaviour of $g_{\mu}^{(\alpha)}(z)$ at the origin, we need to determine the exact expression of this constant term. For such a purpose, we will proceed by induction again. Consider first the pure case $\mathrm{I}^{k}(k=q)$, where $\alpha^{\prime}=\alpha-k$. From (3.9) and (3.10), we have

$$
g_{\mu}^{(\alpha)}(z)=\left|\begin{array}{ccc}
L_{m_{1}}^{\left(\alpha^{\prime}\right)}(-z) & \ldots & L_{m_{k}}^{\left(\alpha^{\prime}\right)}(-z) \\
\vdots & \ddots & \vdots \\
L_{m_{1}-k+1}^{\left(\alpha^{\prime}+k-1\right)}(-z) & \ldots & L_{m_{k}-k+1}^{\left(\alpha^{\prime}+k-1\right)}(-z)
\end{array}\right| .
$$

On using ${ }^{34}$

$$
L_{m}^{(\alpha)}(0)=\frac{(\alpha+1)_{m}}{m !}
$$

we easily get

$$
\begin{aligned}
& g_{\mu}^{(\alpha)}(0)= \frac{\left(\alpha^{\prime}+k\right)_{m_{1}-k+1} \cdots\left(\alpha^{\prime}+k\right)_{m_{k}-k+1}}{m_{1} ! \cdots m_{k} !} \\
& \times\left|\begin{array}{ccc}
\left(\alpha^{\prime}+1\right) \cdots\left(\alpha^{\prime}+k-1\right) & \ldots & \left(\alpha^{\prime}+1\right) \cdots\left(\alpha^{\prime}+k-1\right) \\
\left(\alpha^{\prime}+2\right) \cdots\left(\alpha^{\prime}+k-1\right) m_{1} & \cdots & \left(\alpha^{\prime}+2\right) \cdots\left(\alpha^{\prime}+k-1\right) m_{k} \\
\vdots & \ddots & \vdots \\
m_{1}\left(m_{1}-1\right) \cdots\left(m_{1}-k+2\right) & \ldots & m_{k}\left(m_{k}-1\right) \cdots\left(m_{k}-k+2\right)
\end{array}\right|,
\end{aligned}
$$

that is (see Eq. (3.14)),

$$
\begin{aligned}
g_{\mu}^{(\alpha)}(0) & =\frac{\left(\alpha^{\prime}+1\right)_{m_{1}} \cdots\left(\alpha^{\prime}+k\right)_{m_{k}-k+1}}{m_{1} ! \cdots m_{k} !} \Delta\left(m_{1}, \ldots, m_{k}\right) \\
& =\frac{(\alpha-k+1)_{m_{1}} \cdots(\alpha)_{m_{k}-k+1}}{m_{1} ! \cdots m_{k} !} \Delta\left(m_{1}, \ldots, m_{k}\right) .
\end{aligned}
$$

Consider now the first mixed case $\mathrm{I}^{k-1} \mathrm{II}(k=q+1)$, where $\alpha^{\prime}=\alpha-k+2 .{ }^{31}$ We then get

$$
g_{\mu}^{(\alpha)}(z)=\left|\begin{array}{cccc}
L_{m_{1}}^{\left(\alpha^{\prime}\right)}(-z) & \ldots & L_{m_{k-1}}^{\left(\alpha^{\prime}\right)}(-z) & z^{k-1} L_{m_{k}}^{\left(-\alpha^{\prime}\right)}(z) \\
\vdots & \ddots & \vdots & \vdots \\
L_{m_{1}-k+2}^{\left(\alpha^{\prime}+k-2\right)}(-z) & \ldots & L_{m_{k-1}-k+2}^{\left(\alpha^{\prime}+k-2\right)}(-z) & \left(m_{k}+1\right)_{k-2} z L_{m_{k}+k-2}^{\left(-\alpha^{\prime}-k+2\right)}(z) \\
L_{m_{1}-k+1}^{\left(\alpha^{\prime}+k-1\right)}(-z) & \ldots & L_{m_{k-1}-k+1}^{\left(\alpha^{\prime}+k-1\right)}(-z) & \left(m_{k}+1\right)_{k-1} L_{m_{k}+k-1}^{\left(-\alpha^{\prime}-k+1\right)}(z)
\end{array}\right|,
$$


which leads to

$$
g_{\mu}^{(\alpha)}(0)=\left(m_{k}+1\right)_{k-1} L_{m_{k}+k-1}^{\left(-\alpha^{\prime}-k+1\right)}(0)\left|\begin{array}{ccc}
L_{m_{1}}^{\left(\alpha^{\prime}\right)}(0) & \ldots & L_{m_{k-1}}^{\left(\alpha^{\prime}\right)}(0) \\
\vdots & \ddots & \vdots \\
L_{m_{1}-k+2}^{\left(\alpha^{\prime}+k-2\right)}(0) & \ldots & L_{m_{k-1}-k+2}^{\left(\alpha^{\prime}+k-2\right)}(0)
\end{array}\right|
$$

The right-hand member determinant is exactly the constant term of the $g$ polynomial in the pure case $\mathrm{I}^{k-1}$ and we deduce from the previous result that

$$
\begin{aligned}
g_{\mu}^{(\alpha)}(0)= & \frac{\left(\alpha^{\prime}+1\right)_{m_{1}} \cdots\left(\alpha^{\prime}+k-1\right)_{m_{k-1}-k+2}}{m_{1} ! \cdots m_{k-1} !} \Delta\left(m_{1}, \ldots, m_{k-1}\right) \\
& \times\left(m_{k}+1\right)_{k-1}(-1)^{m_{k}+k-1} \frac{\left(\alpha^{\prime}-m_{k}\right)_{m_{k}+k-1}}{\left(m_{k}+k-1\right) !}
\end{aligned}
$$

or

$$
\begin{aligned}
g_{\mu}^{(\alpha)}(0)= & (-1)^{m_{k}+k-1} \frac{\left(\alpha^{\prime}+1\right)_{m_{1}} \cdots\left(\alpha^{\prime}+k-1\right)_{m_{k-1}-k+2}\left(\alpha^{\prime}-m_{k}\right)_{m_{k}+k-1}}{m_{1} ! \cdots m_{k} !} \\
& \times \Delta\left(m_{1}, \ldots, m_{k-1}\right) \\
= & (-1)^{m_{k}+k-1} \frac{(\alpha-k+3)_{m_{1}} \cdots(\alpha+1)_{m_{k-1}-k+2}\left(\alpha-k+2-m_{k}\right)_{m_{k}+k-1}}{m_{1} ! \cdots m_{k} !} \\
& \times \Delta\left(m_{1}, \ldots, m_{k-1}\right) .
\end{aligned}
$$

For the general mixed case $\mathrm{I}^{q} \mathrm{II}^{k-q}$, we now make the following hypothesis:

$$
\begin{aligned}
g_{\mu}^{(\alpha)}(0)= & (-1)^{\sigma} \frac{\Delta\left(m_{1}, \ldots, m_{q}\right) \Delta\left(m_{q+1}, \ldots, m_{k}\right)}{m_{1} ! \cdots m_{k} !} \\
& \times\left((\alpha+k-2 q+1)_{m_{1}} \cdots(\alpha+k-q)_{m_{q}-q+1}\right) \\
& \times\left(\left(\alpha+k-2 q-m_{q+1}\right)_{m_{q+1}+q} \cdots\left(\alpha+k-2 q-m_{k}\right)_{m_{k}+2 q-k+1}\right),
\end{aligned}
$$

with $\sigma$ defined in Eq. (3.13), and prove it by induction over $k$.

This conjecture is verified in the $k=1$ and $k=2$ cases. Since it is also valid for the two previous examples ( $k-q=0$ or 1 ), it only remains to prove it whenever $k-q \geq 2$. In such cases, the last two seed functions $\phi_{k-1}$ and $\phi_{k}$, used in $k$ th-order SUSYQM, are necessarily of type II. Suppose that Eq. (4.23) is verified for $k^{\prime}<k$. We may therefore write

$$
\begin{aligned}
& g_{\mu^{\prime \prime}}^{(\alpha+2)}(0)=(-1)^{\sigma^{\prime \prime}} \frac{\Delta\left(m_{1}, \ldots, m_{q}\right) \Delta\left(m_{q+1}, \ldots, m_{k-2}\right)}{m_{1} ! \cdots m_{k-2} !} \\
& \quad \times\left((\alpha+k-2 q+1)_{m_{1}} \cdots(\alpha+k-q)_{m_{q}-q+1}\right) \\
& \quad \times\left(\left(\alpha+k-2 q-m_{q+1}\right)_{m_{q+1}+q} \cdots\left(\alpha+k-2 q-m_{k-2}\right)_{m_{k-2}+2 q-k+3}\right), \\
& \quad \times\left((\alpha+k-2 q+1)_{m_{1}} \cdots(\alpha+k-q)_{m_{q}-q+1}\right) \\
& \quad \times\left(\left(\alpha+k-2 q-m_{q+1}\right)_{m_{q+1}+q} \cdots\left(\alpha+k-2 q-m_{k-1}\right)_{m_{k-1}+2 q-k+2}\right),
\end{aligned}
$$


and

$$
\begin{aligned}
& g_{\bar{\mu}^{\prime}}^{(\alpha+1)}(0)=(-1)^{\bar{\sigma}^{\prime}} \frac{\Delta\left(m_{1}, \ldots, m_{q}\right) \Delta\left(m_{q+1}, \ldots, m_{k-2}, m_{k}\right)}{m_{1} ! \cdots m_{k-2} ! m_{k} !} \\
& \quad \times\left((\alpha+k-2 q+1)_{m_{1}} \cdots(\alpha+k-q)_{m_{q}-q+1}\right) \\
& \quad \times\left(\left(\alpha+k-2 q-m_{q+1}\right)_{m_{q+1}+q} \cdots\left(\alpha+k-2 q-m_{k-2}\right)_{m_{k-2}+2 q-k+3}\right), \\
& \quad \times\left(\alpha+k-2 q-m_{k}\right)_{m_{k}+2 q-k+2},
\end{aligned}
$$

with

$$
\left\{\begin{array}{c}
\sigma^{\prime}=\sum_{l=q+1}^{k-1} m_{l}+q(k-q-1), \\
\sigma^{\prime \prime}=\sum_{l=q+1}^{k-2} m_{l}+q(k-q-2), \\
\bar{\sigma}^{\prime}=\sum_{l=q+1}^{k-2} m_{l}+m_{k}+q(k-q-1) .
\end{array}\right.
$$

From Eq. (4.14), we obtain the recurrence relation

$$
g_{\mu}^{(\alpha)}(0)=\frac{m_{k}-m_{k-1}}{\alpha+1} g_{\mu^{\prime}}^{(\alpha+1)}(0) g_{\bar{\mu}^{\prime}}^{(\alpha+1)}(0) / g_{\mu^{\prime \prime}}^{(\alpha+2)}(0),
$$

which combined with the previous expressions yields

$$
\begin{aligned}
g_{\mu}^{(\alpha)}(0)= & \frac{(-1)^{\sigma^{\prime}+\bar{\sigma}^{\prime}-\sigma^{\prime \prime}}\left(m_{k}-m_{k-1}\right)}{(\alpha+1) m_{1} ! \cdots m_{k} !} \\
& \times \frac{\Delta\left(m_{1}, \ldots, m_{q}\right) \Delta\left(m_{q+1}, \ldots, m_{k-1}\right) \Delta\left(m_{q+1}, \ldots, m_{k-2}, m_{k}\right)}{\Delta\left(m_{q+1}, \ldots, m_{k-2}\right)} \\
& \times\left((\alpha+k-2 q+1)_{m_{1}} \cdots(\alpha+k-q)_{m_{q}-q+1}\right) \\
& \times\left(\left(\alpha+k-2 q-m_{q+1}\right)_{m_{q+1}+q} \cdots\left(\alpha+k-2 q-m_{k-2}\right)_{m_{k-2}+2 q-k+3}\right) \\
& \times\left(\alpha+k-2 q-m_{k-1}\right)_{m_{k-1}+2 q-k+2}\left(\alpha+k-2 q-m_{k}\right)_{m_{k}+2 q-k+2} .
\end{aligned}
$$

Here we note that

$$
\begin{gathered}
\sigma^{\prime}+\bar{\sigma}^{\prime}-\sigma^{\prime \prime}=\sum_{l=q+1}^{k-2} m_{l}+m_{k-1}+m_{k}+q(k-q)=\sigma, \\
\frac{\left(\alpha+k-2 q-m_{k}\right)_{m_{k}+2 q-k+2}}{\alpha+1}=\left(\alpha+k-2 q-m_{k}\right)_{m_{k}+2 q-k+1},
\end{gathered}
$$

and

$$
\begin{aligned}
& \left(m_{k}-m_{k-1}\right) \frac{\Delta\left(m_{q+1}, \ldots, m_{k-1}\right) \Delta\left(m_{q+1}, \ldots, m_{k-2}, m_{k}\right)}{\Delta\left(m_{q+1}, \ldots, m_{k-2}\right)} \\
& =\left(m_{k}-m_{k-1}\right)\left(\prod_{i=q+1}^{k-2} \prod_{j=i+1}^{k-1}\left(m_{j}-m_{i}\right)\right)\left(\prod_{i=q+1}^{k-2}\left(m_{k}-m_{i}\right)\right) \\
& =\prod_{i=q+1}^{k-1} \prod_{j=i+1}^{k}\left(m_{j}-m_{i}\right)=\Delta\left(m_{q+1}, \ldots, m_{k}\right) .
\end{aligned}
$$

The final result for $g_{\mu}^{(\alpha)}(0)$ is therefore given by Eq. (4.23), showing that the conjecture is still verified for $k$, which ends its proof. 
Let us observe that in terms of $\alpha^{\prime}$, Eq. (4.23) can be rewritten as

$$
\begin{aligned}
g_{\mu}^{(\alpha)}(0)= & (-1)^{\sigma} \frac{\Delta\left(m_{1}, \ldots, m_{q}\right) \Delta\left(m_{q+1}, \ldots, m_{k}\right)}{m_{1} ! \cdots m_{k} !} \\
& \times\left(\left(\alpha^{\prime}+1\right)_{m_{1}} \cdots\left(\alpha^{\prime}+q\right)_{m_{q}-q+1}\right) \\
& \times\left(\left(\alpha^{\prime}-m_{q+1}\right)_{m_{q+1}+q} \cdots\left(\alpha^{\prime}-m_{k}\right)_{m_{k}+2 q-k+1}\right) .
\end{aligned}
$$

Since the angular momentum $l^{\prime}$ in the starting potential $V^{(1)}(x)$ is necessarily nonnegative, the inequality $\alpha^{\prime}>-1 / 2$ is fulfilled. Furthermore, we have assumed $0<m_{1}<\cdots<m_{q}$ and $0<m_{q}+1$ $<\cdots<m_{k}<\alpha^{\prime}$. As a consequence, the two Vandermonde determinants in Eq. (4.33) are positive and

$$
\left(\alpha^{\prime}+1\right)_{m_{1}} \cdots\left(\alpha^{\prime}+q\right)_{m_{q}-q+1}\left(\alpha^{\prime}-m_{q+1}\right)_{m_{q+1}+q} \cdots\left(\alpha^{\prime}-m_{k}\right)_{m_{k}+2 q-k+1}>0 .
$$

We conclude that the sign of $g_{\mu}^{(\alpha)}(0)$ is given by $(-1)^{\sigma}$, hence is identical with the one of $\mathcal{C}_{\mu}^{(\alpha)}$ (see Eq. (3.13)). The combination of this result with the disconjugacy theorem of Sec. II ensures the regularity of the extended potential $V^{(2)}(x)$.

\section{CONCLUSION}

In the present work, we have established some further properties of the function $g_{\mu}^{(\alpha)}(z)$ appearing in the denominator of rationally extended isotonic oscillator (or radial oscillator) potentials constructed in $k$ th-order SUSYQM ${ }^{31}$ or using the multistep Darboux-Bäcklund transformation method. ${ }^{32}$ We have first confirmed that $g_{\mu}^{(\alpha)}(z)$ cannot have any pole at the origin and is a polynomial with non-zero constant term, a property that was conjectured in Ref. 31. We have then determined the value of this constant term by induction over $k$ and shown that it has the same sign as the coefficient of the previously determined highest degree term $z^{\mu}{ }^{31}$

These results have been combined with the disconjugacy theorem applied to Schrödinger equation $^{32}$ to prove the absence of zeros of $g_{\mu}^{(\alpha)}(z)$ on the positive half-line and, therefore, the regularity of the corresponding rationally extended potential. This has provided a demonstration of the latter property, which is more explicit than that already given in Ref. 32.

Our work has illustrated the power of the disconjugacy properties of second-order differential equations of Schrödinger type to check the regularity of rational extensions of well-known quantum potentials. Other examples of application might be found in cases where no direct information on the denominator zeros is available. This will be the object of further investigations.

${ }^{1}$ D. Gómez-Ullate, N. Kamran, and R. Milson, "The Darboux transformation and algebraic deformations of shape-invariant potentials,” J. Phys. A 37, 1789 (2004); e-print arXiv:quant-ph/0308062.

${ }^{2}$ D. Gómez-Ullate, N. Kamran, and R. Milson, "Supersymmetry and algebraic Darboux transformations," J. Phys. A 37, 10065 (2004); e-print arXiv:nlin.SI/0402.052.

${ }^{3}$ D. Gómez-Ullate, N. Kamran, and R. Milson, "An extended class of orthogonal polynomials defined by a Sturm-Liouville problem,” J. Math. Anal. Appl. 359, 352 (2009); e-print arXiv:0807.3939.

${ }^{4}$ D. Gómez-Ullate, N. Kamran, and R. Milson, “An extension of Bochner's problem: Exceptional invariant subspaces,” J. Approx. Theory 162, 987 (2010); e-print arXiv:0805.3376.

${ }^{5}$ C. Quesne, "Exceptional orthogonal polynomials, exactly solvable potentials and supersymmetry," J. Phys. A 41, 392001 (2008); e-print arXiv:0807.4087.

${ }^{6}$ L. E. Gendenshtein, "Derivation of exact spectra of the Schrödinger equation by means of supersymmetry," JETP Lett. 38, 356 (1983).

${ }^{7}$ F. Cooper, A. Khare, and U. Sukhatme, "Supersymmetry and quantum mechanics," Phys. Rep. 251, 267 (1995); e-print arXiv:hep-th/9405029.

${ }^{8}$ J. F. Cariñena and A. Ramos, "Shape-invariant potentials depending on $n$ parameters transformed by translation," J. Phys. A 33, 3467 (2000); e-print arXiv:hep-th/0003266.

${ }^{9}$ B. Bagchi, C. Quesne, and R. Roychoudhury, "Isospectrality of conventional and new extended potentials, second-order supersymmetry and role of $\mathcal{P} \mathcal{T}$ symmetry," Pramana, J. Phys. 73, 337 (2009); e-print arXiv:0812.1488.

${ }^{10} \mathrm{C}$. Quesne, "Solvable rational potentials and exceptional orthogonal polynomials in supersymmetric quantum mechanics," SIGMA 5, 084 (2009); e-print arXiv:0906.2331.

${ }^{11} \mathrm{~S}$. Odake and R. Sasaki, "Infinitely many shape invariant potentials and new orthogonal polynomials," Phys. Lett. B 679, 414 (2009); e-print arXiv:0906.0142. 
${ }^{12}$ S. Odake and R. Sasaki, “Another set of infinitely many exceptional $\left(X_{\ell}\right)$ Laguerre polynomials," Phys. Lett. B 684, 173 (2010); e-print arXiv:0911.3442.

${ }^{13} \mathrm{~S}$. Odake and R. Sasaki, "Infinitely many shape-invariant potentials and cubic identities of the Laguerre and Jacobi polynomials," J. Math. Phys. 51, 053513 (2010); e-print arXiv:0911.1585.

${ }^{14}$ C.-L. Ho, S. Odake, and R. Sasaki, "Properties of the exceptional $\left(X_{\ell}\right)$ Laguerre and Jacobi polynomials," SIGMA 7, 107 (2011); e-print arXiv:0912.5447.

${ }^{15}$ R. Sasaki, S. Tsujimoto, and A. Zhedanov, "Exceptional Laguerre and Jacobi polynomials and the corresponding potentials through Darboux-Crum transformations," J. Phys. A 43, 315204 (2010); e-print arXiv:1004.4711.

${ }^{16}$ D. Gómez-Ullate, N. Kamran, and R. Milson, "Exceptional orthogonal polynomials and the Darboux transformation," J. Phys. A 43, 434016 (2010); e-print arXiv:1002.2666.

${ }^{17}$ D. Gómez-Ullate, N. Kamran, and R. Milson, "On orthogonal polynomials spanning a non-standard flag,” Contemp. Math. 563, 51 (2012); e-print arXiv:1101.5584.

${ }^{18}$ Y. Grandati, "Solvable rational extensions of the isotonic oscillator," Ann. Phys. (N.Y.) 326, 2074 (2011); e-print arXiv:1101.0055.

${ }^{19}$ C.-L. Ho, "Prepotential approach to solvable rational potentials and exceptional orthogonal polynomials," Prog. Theor. Phys. 126, 185 (2011); e-print arXiv:1104.3511.

${ }^{20}$ S. Yu. Dubov, V. M. Eleonskii, and N. E. Kulagin, "Equidistant spectra of anharmonic oscillators," Sov. Phys. JETP 75, 446 (1992).

${ }^{21}$ S. Yu. Dubov, V. M. Eleonskii, and N. E. Kulagin, "Equidistant spectra of anharmonic oscillators," Chaos 4, 47 (1994).

${ }^{22}$ V. G. Bagrov and B. F. Samsonov, "Darboux transformation, factorization, and supersymmetry in one-dimensional quantum mechanics," Theor. Math. Phys. 104, 1051 (1995).

${ }^{23}$ B. F. Samsonov, "New features in supersymmetry breakdown in quantum mechanics," Mod. Phys. Lett. A 11, 1563 (1996); e-print arXiv:quant-ph/9611012.

${ }^{24}$ G. Junker and P. Roy, "Conditionally exactly solvable problems and non-linear algebras," Phys. Lett. A 232, 155 (1997).

${ }^{25}$ G. Junker and P. Roy, "Conditionally exactly solvable potentials: A supersymmetric construction method," Ann. Phys. (N.Y.) 270, 155 (1998); e-print arXiv:quant-ph/9803024.

${ }^{26}$ J. F. Cariñena, A. M. Perelomov, M. F. Rañada, and M. Santander, "A quantum exactly solvable nonlinear oscillator related to the isotonic oscillator," J. Phys. A 41, 085301 (2008); e-print arXiv:0711.4899.

${ }^{27}$ J. M. Fellows and R. A. Smith, "Factorization solution of a family of quantum nonlinear oscillators," J. Phys. A 42, 335303 (2009).

${ }^{28}$ D. Gómez-Ullate, N. Kamran, and R. Milson, "Two-step Darboux transformations and exceptional Laguerre polynomials," J. Math. Anal. Appl. 387, 410 (2012); e-print arXiv:1103.5724.

${ }^{29}$ S. Odake and R. Sasaki, "Exactly solvable quantum mechanics and infinite families of multi-indexed orthogonal polynomials," Phys. Lett. B 702, 164 (2011); e-print arXiv:1105.0508.

${ }^{30}$ C. Quesne, "Higher-order SUSY, exactly solvable potentials, and exceptional orthogonal polynomials," Mod. Phys. Lett. A 26, 1843 (2011); e-print arXiv:1106.1990.

${ }^{31}$ C. Quesne, "Rationally-extended radial oscillators and Laguerre exceptional orthogonal polynomials in $k$ th-order SUSYQM,” Int. J. Mod. Phys. A 26, 5337 (2011); e-print arXiv:1110.3958.

${ }^{32}$ Y. Grandati, "Multistep DBT and regular rational extensions of the isotonic oscillator," Ann. Phys. (N.Y.) 327, 2411 (2012); e-print arXiv:1108.4503.

${ }^{33}$ D. Gómez-Ullate, N. Kamran, and R. Milson, "A conjecture on exceptional orthogonal polynomials," Found. Comput. Math. (2012); e-print arXiv:1203.6857.

${ }^{34}$ A. Erdélyi, W. Magnus, F. Oberhettinger, and F. G. Tricomi, Higher Transcendental Functions (McGraw-Hill, New York, 1953).

${ }^{35}$ G. Szegö, Orthogonal Polynomials (American Mathematical Society, Providence, RI, 1939).

${ }^{36}$ P. Hartman, Ordinary Differential Equations (John Wiley, New York, 1964).

${ }^{37}$ W. A. Coppel, Disconjugacy (Springer, Berlin, 1971).

${ }^{38}$ M. Böcher, Leçons sur les méthodes de Sturm (Gauthier-Villars, Paris, 1917).

${ }^{39}$ A. A. Andrianov, M. V. Ioffe, and V. Spiridonov, "Higher-derivative supersymmetry and the Witten index," Phys. Lett. A 174, 273 (1993); e-print arXiv:hep-th/9303005.

${ }^{40}$ A. A. Andrianov, M. V. Ioffe, F. Cannata, and J.-P. Dedonder, "Second order derivative supersymmetry, $q$ deformations and the scattering problem,” Int. J. Mod. Phys. A 10, 2683 (1995); e-print arXiv:hep-th/9404061.

${ }^{41}$ D. J. Fernández C. and N. Fernández-García, "Higher-order supersymmetric quantum mechanics," AIP Conf. Proc. 744, 236 (2004); e-print arXiv:quant-ph/0502098.

${ }^{42}$ M. M. Crum, “Associated Sturm-Liouville systems,” Quart. J. Math. Oxford Ser. (2) 6, 121 (1955).

${ }^{43}$ T. Muir, A Treatise on the Theory of Determinants (Dover, New York, 1960) (revised and enlarged by W. H. Metzler).

${ }^{44}$ A. Messiah, Mécanique Quantique (Dunod, Paris, 1969), Vol. 1. 